\title{
Intake patterns and dietary associations of soya protein consumption in adults and children in the Canadian Community Health Survey, Cycle 2.2
}

\author{
Adriana N. Mudryj ${ }^{1}$, Harold M. Aukema ${ }^{1}$ and Nancy $\mathrm{Yu}^{2,3 *}$ \\ ${ }^{1}$ Human Nutritional Sciences, University of Manitoba, Winnipeg, MB R3T 2N2, Canada \\ ${ }^{2}$ Community Health Sciences, University of Manitoba, Winnipeg, MB R3E OT6, Canada \\ ${ }^{3}$ Manitoba Health, Winnipeg, MB R3B 3M9, Canada \\ (Submitted 10 July 2014 - Final revision received 29 September 2014 - Accepted 16 October 2014 - First published online 6 January 2015)
}

\section{Abstract}

Soya foods are one of the recommended alternatives to meat in many dietary guidelines. While this is expected to increase the intake of some nutrients, potential concerns regarding others have been raised. The purpose of the present study was to examine the prevalence and the association of soya food consumption with nutrient intakes and dietary patterns of Canadians (age $\geq 2$ years). Cross-sectional data from the 2004 Canadian Community Health Survey (Cycle 2.2; n 33218) were used to classify soya consumers and non-consumers. Soya consumers were further divided into two groups based on their soya protein intake. Sample weights were applied and logistic regression analysis was used to explore the association between nutrient intakes and soya consumption, with cultural background, sex, age and economic status being included as covariates. On any given day, 3.3\% ( $n$ 1085) of Canadians consume soya foods, with females, Asian Canadians and adults with post-secondary education being more likely to be soya consumers. As a whole, adolescent and adult respondents who had consumed at least one soya food during their $24 \mathrm{~h}$ dietary recall had higher energy intakes, as well as increased intakes of nutrients such as protein, fibre, vitamin $\mathrm{C}$, vitamin $\mathrm{B}_{6}$, naturally occurring folate, thiamin, $\mathrm{Ca}, \mathrm{P}, \mathrm{Mg}, \mathrm{PUFA}$, Fe and $\mathrm{K}$ and lowered intakes of saturated fat. These data indicate that soya food consumption is associated with improved diet quality of Canadians. However, future research is necessary to investigate the association between increased energy intake and soya consumption.

Key words: Soya protein: Health surveys: Dietary intakes: Canadian Community Health Survey

Soyabeans are rich in nutrients such as $\mathrm{Ca}$, Fe, riboflavin and $\mathrm{K}$, and contain compounds such as isoflavones and lecithins that have been associated with a reduction in chronic disease and an improvement in bone health, particularly in women $^{(1-6)}$. In addition, soya protein contains adequate quantities of essential amino acids ${ }^{(2)}$. In 1999, the US Food and Drug Administration (FDA) approved a food-labelling health claim for soya foods based on their conclusion that daily consumption of $25 \mathrm{~g}$ soya protein coupled with a low-fat diet may reduce the risk of CVD by lowering blood cholesterol levels ${ }^{(7)}$. Asian populations who consume soya as a staple food have been shown to have a reduced risk of CVD compared with their Western counterparts ${ }^{(8-10)}$. A decreased risk of breast cancer in pre-menopausal Chinese women who were high consumers of soya foods was first reported by Lee et $a l^{(11)}$. More recently, a study that examined the risk of breast cancer in Chinese women has found that women with high soya isoflavone intakes have a decreased risk of breast cancer mortality, while women with high soya protein intakes have a lower risk of breast cancer than those with lower consumption ${ }^{(12)}$. Although research involving soya consumption and breast cancer has been extensive, overall results are not conclusive. In addition, the mechanism between intake of soya and reduced risk of breast cancer remains unclear ${ }^{(13)}$.

Phyto-oestrogens in soya and soya protein have been positively associated with bone-protective effects in women, and with bone and $\mathrm{Ca}$ balance in postmenopausal women, respectively ${ }^{(2,14)}$. Consumption of soya proteins may reduce $\mathrm{Ca}$ excretion due to their lower sulphur amino acid content $^{(15)}$. In addition, intake of soya isoflavones has been found to significantly increase bone mineral density in periand postmenopausal women ${ }^{(16)}$, possibly due to their interaction with vitamin $\mathrm{D}$ in stimulating bone formation and reducing resorption ${ }^{(17)}$. Although many studies have been conducted to investigate the beneficial effects of soya food

Abbreviations: CCHS 2.2, Canadian Community Health Survey, Cycle 2.2; CNF, Canadian Nutrient File; EPIC, European Prospective Investigation into Cancer and Nutrition; FDA, US Food and Drug Administration; NHANES, National Health and Nutrition Examination Survey; USDA, United States Department of Agriculture. 
consumption on the prevention of bone loss or osteoporosis in postmenopausal women due to soya isoflavones, results remain inconclusive, and further randomised controlled trials are necessary to better elucidate the relationship between isoflavones and bone loss ${ }^{(18-20)}$.

Studies have shown that replacement of meat and dairy products with soya products may improve diet quality. For example, replacing a 3-ounce $(85 \mathrm{~g})$ patty made from ground beef with a soya patty leads to a reduction of $12 \mathrm{~g}$ fat and $5 \mathrm{~g}$ saturated fat. Similarly, replacing a cup of whole milk with a cup of soya milk can reduce the intake of fat and saturated fat by 4 and $4.5 \mathrm{~g}$, respectively. The partial replacement of traditional protein ingredients with tofu led to enhanced nutritional quality recipes used in American preschools. When tofu replaced cheese, the amount of fat, cholesterol, $\mathrm{Na}$ and energy was reduced; when it replaced beef, the amount of fat and cholesterol decreased and when it replaced egg or chicken, the amount of cholesterol decreased. When cheese or egg was replaced by tofu, children ate more of the new dish but when beef or chicken was replaced, they ate more of the original dish ${ }^{(21)}$. A simulation analysis in which servings of meat were replaced with tofu has shown that this replacement would increase the intakes of nutrients such as folate, $\mathrm{Fe}, \mathrm{Ca}$ and $\mathrm{Mg}$ by $>10 \%$ and lower the intakes of saturated fat, cholesterol, and vitamins $\mathrm{B}_{6}$ and $\mathrm{B}_{12}$. If both meat and dairy products were replaced with soya equivalents (i.e. tofu or soya milk), intakes of fibre, folate, vitamin $\mathrm{K}, \mathrm{Fe}$, $\mathrm{Ca}$ and $\mathrm{Mg}$ would be expected to increase and intakes of saturated fat and cholesterol would decrease ${ }^{(22)}$. However, concerns regarding compromised nutrient intake in soya consumers have also been raised. With these substitutions, intakes of protein, riboflavin, and vitamins $\mathrm{B}_{6}$ and $\mathrm{B}_{12}$ would be lowered ${ }^{(21,22)}$

Previously published data from the European Prospective Investigation into Cancer and Nutrition (EPIC) study have described soya consumption patterns in Europe ${ }^{(23)}$. Similarly, Messina and colleagues ${ }^{(9,24)}$ analysed intake of soya protein in four Asian nations. However, there are limited data on soya eating habits in North America. Recent reports have suggested that Canadian children do not receive adequate intakes of vitamin $\mathrm{D}$ and $\mathrm{Ca}$, and that many adults do not meet the requirements for intakes of $\mathrm{Mg}, \mathrm{Ca}, \mathrm{K}$, fibre and vitamin $\mathrm{D}^{(25,26)}$, with all these nutrients being present in high amounts in soya foods. The purpose of the present study was to describe the demographic characteristics of Canadian soya consumers, and to compare the dietary patterns and nutrient profiles of soya consumers with those of non-consumers.

\section{Methods}

The present study used the data from the Canadian Community Health Survey, Cycle 2.2 (CCHS 2.2) conducted by Statistics Canada, and methods similar to those used in the previous analysis of bean, pea and lentil consumption patterns ${ }^{(27)}$. The CCHS 2.2 was completed in 2004 and targeted respondents from all age groups living in the ten provinces. The main objectives were to collect information on the nutritional status of Canadians and to estimate the distribution of dietary intakes in terms of foods, food groups, dietary supplements, nutrients and eating patterns among a representative sample of Canadians at national and provincial levels using a $24 \mathrm{~h}$ dietary recall. A total of 35107 adults and children completed the initial $24 \mathrm{~h}$ dietary recall. Following this, a subsample of 10786 completed a second recall $3-10 \mathrm{~d}$ later. The $24 \mathrm{~h}$ dietary recalls were collected primarily by face-to-face interviews by trained interviewers $^{(28,29)}$. Further details on the methods used in the CCHS 2.2 are available on the Health Canada Website ${ }^{(28)}$.

Although the main outcome variable was dietary intake, the CCHS 2.2 also collected physical measurements, demographic characteristics and socio-economic data from respondents. The main demographic variables that were examined in the present analysis included sex, age, provincial location and cultural background. Income and education were also examined (for those aged $\geq 19$ years), splitting the respondents into four groups based on their household income adequacy or highest level of education attained ${ }^{(28,29)}$.

Data for the present analysis were limited to Canadians aged $\geq 2$ years ( $n$ 33941). Children under 2 years of age were excluded due to the dramatic change in food sources during the first 2 years of life, and the fact that conventional foods (rather than breast milk-based diets) are introduced into the diet about 2 years of age ${ }^{(30,31)}$. Although second-day consumption levels were examined, they only assessed a small subset of the original survey, thus nutrient intake assessments were limited to $1 \mathrm{~d}$ recalls only. Respondents who did not report consuming any food, only reported consuming breast milk or whose recalls were considered to be unreliable according to Health Canada were excluded, leaving a total sample size of 33218. Pregnant and breast-feeding women were included in the present study. Although data on vitamin and mineral supplementation were collected in the CCHS 2.2, the present analysis considers nutrient intakes from food only, which were obtained using values from the Canadian Nutrient File $(\mathrm{CNF})^{(32)}$. Consumers were identified as individuals who had reported eating at least one soya product during their recall period. Food sources included soyabeans, soya beverages, soya flour, soya protein powders and isolates, soya bread, tofu and other fermented products, soya-based dairy products (e.g. cheese and ice cream), and soya-based meat alternatives (e.g. patties or wieners). Respondents who reported soya sauce and soya-based margarines as their only soya consumption were not considered as soya consumers. Thus, soya sauce and soya-based margarines were excluded. The amount of soya protein per $100 \mathrm{~g}$ of soya product was calculated using the CNF, the $2001 \mathrm{~b}$ recipe database and the United States Department of Agriculture (USDA) Food and Nutrient Database for Dietary Studies, version 1.0. The CNF is a continuously updated bilingual database that contains information on 150 nutrients in over 5807 foods, which was utilised by Health Canada to report the nutrient content of the foods reported by the respondents of the CCHS 2.2. The CNF also contains data from the USDA Food and Nutrient Database for Dietary Studies, version 1.0, for corresponding foods (in particular for mixed dishes) as well as comprises modified data that reflect Canadian food supply and unique Canadian dishes ${ }^{(32)}$. Further details on both the CNF database 
(including information on serving size) and the USDA Food and Nutrient Database are available on their respective web sites $^{(32-34)}$. Food group intake data were obtained from the Canada's Food Guide File that contained previously calculated food group servings for each survey respondent ${ }^{(29,33)}$.

Soya consumers were divided into three age groups, 2-8 years ( $n$ 128), 9-18 years $(n$ 226) and $\geq 19$ years ( $n$ 731), totalling 1085 soya consumers overall. Consumers in each age group were then further divided into two groups based on the median level of soya protein consumed (in $\mathrm{g}$ ) in their respective group, resulting in two equal-sized groups of consumers. Soya consumers aged 2-8 years were split into two groups of sixty-four each, those aged 9-18 years into two groups of 113 each, and those aged $\geq 19$ years into two groups of 366 and 365 each. Respondents who consumed soya protein in amounts less than the median value were referred to as 'low consumers', while those who ate soya protein above the median level were grouped as 'high consumers'. Logistic regression analysis was used to determine whether any demographic variables (sex, age, cultural background, province of residence, income adequacy and education level) increased the likelihood of being classified as a soya consumer, and OR were calculated. Results are reported as OR with $95 \%$ CI. Data for macronutrient and micronutrient intakes were expressed as absolute values, as well as quantity per $4184 \mathrm{~kJ}(1000 \mathrm{kcal})$. General linear models were used to analyse macronutrient and micronutrient intakes and to compare nutrient intakes and other variables between non-consumers and consumers as well as between non-consumers and consumers at each of the two levels of consumption. In addition, similar analyses were conducted for each of the food groups using the data from the CCHS's Canada Food Guide file. The significance level was set at $P<0.05$ for differences between groups and $0.05<P<0.10$ for trends. It is important to note that because the CCHS 2.2 is a population-based survey, results represent population estimates and do not represent individual or chronic dietary exposure.

The bootstrapping method was used in all the data analyses of the present study. This approximation technique is recommended by Statistics Canada for use with the CCHS 2.2 to estimate standard errors, coefficients of variation and CI. Using SUDAAN software, bootstrapping was used to estimate distribution from a sample's statistics and involves the selection of random samples known as replicates and the calculation of the variation in estimates from replicate to replicate ${ }^{(28)}$. All analyses were performed using PASW SPSS Statistics, version 18 (IBM) and SUDAAN Statistical Analysis Software Package, version 10.0.1 (RTI International).

\section{Results}

\section{Demographics of Canadian soya consumers}

The median age of soya consumers and non-consumers was very similar among all the age groups: for 2- to 8-year-olds, it was 5 years; for 9- to 18-year-olds, it was 15 years for soya consumers and 14 years for non-consumers; for adults $\geq 19$ years, it was 47 years for soya consumers and 52 years for non-consumers. Adult females were significantly more likely to consume soya products than male adults $(P<0.05)$, yet females consumed less soya protein overall, regardless of age. Geographically, British Columbia had higher proportions of both paediatric and adult soya consumers than did the rest of Canada. In addition, Ontario youth aged 9-18 years were also more likely to be soya consumers. Ethnicity was also an important factor of soya consumption status, with both adult and paediatric Asian Canadian respondents being significantly more likely to consume soya protein than any other ethnic group (Table 1). In adults, income was not a significant factor of soya consumption status; however, respondents who had completed post-secondary education were more likely to be soya consumers than their counterparts with lower education levels.

\section{Food sources}

On any given day, $3 \cdot 3 \%(95 \%$ CI $3 \cdot 1,3 \cdot 5)$ of Canadian adults and youths consume soya products. The main sources of soya protein in the adult Canadian diet were as follows: soya flour; tofu products; soya beverages; soya protein isolate; soyabeans; soya meat substitutes; soyabean soups and fermented products; soya cheese and yogurt; and soya bread or cereal products. Canadian youth data showed similar results in terms of the types of soya foods consumed, with the exception that 9- to 18-year-olds consumed less soya flour and more soya beverages than adults (Fig. 1).

\section{Dietary associations of soya protein consumption}

In children aged 2-8 years, nutrient intakes differed between soya consumers and non-consumers only when expressed relative to energy intake. Respondents at the highest level of soya protein intake consumed more fibre (29\%), Ca (23\%), $\mathrm{Mg}(18 \%), \mathrm{Fe}(14 \%)$ and protein (16\%) per $4184 \mathrm{~kJ}$ (1000 kcal) consumed, and had lower intakes of saturated fat at both levels of soya consumption ( $21 \%$ less in both groups) (Table 2)

In Canadian youths aged 9-18 years, high soya consumers ate more food $(\mathrm{g} ; 21 \%)$ and had higher intakes of fibre $(25 \%)$, protein $(26 \%)$, vitamin $\mathrm{B}_{6}(33 \%)$, naturally occurring folate (43\%), pteroyl(mono)glutamic acid (43\%), Ca (34\%), P (21\%), $\mathrm{Mg}(31 \%), \mathrm{Fe}(24 \%), \mathrm{Zn}(25 \%)$ and K (24\%). When nutrient intakes were calculated relative to total energy intake, vitamin $\mathrm{B}_{6}$, Ca and $\mathrm{Mg}$ remained significantly higher. Soya consumers aged 9-18 years also had higher intakes of fruits and vegetables and meat and alternatives than their non-consuming counterparts (Table 3)

Adult soya consumers in the highest category of soya protein intake consumed 16.5 (SE $2 \cdot 0$ ) g soya protein and had higher intakes of many nutrients. Compared with nonconsumers, high soya consumers had 10\% higher energy intakes $(\mathrm{kJ} / \mathrm{d})$ and consumed a greater amount of food $(\mathrm{g} / \mathrm{d})$ overall. Also, high soya consumers had significantly higher intakes of carbohydrate (9\%), fibre (36\%), PUFA (44\%), linoleic acid (48\%), linolenic acid (32\%), protein (27\%), vitamin C (25\%), thiamin (29\%), vitamin $\mathrm{B}_{6}(26 \%)$, naturally occurring 
Table 1. Demographic characteristics (\% of total Canadian population) of all soya consumers based on $1 \mathrm{~d}$ intakes from the Canadian Community Health Survey, Cycle 2.2, 2004 (Odds ratios and $95 \%$ confidence intervals; mean values of soya protein consumed $(\mathrm{g})$ with their standard errors)

\begin{tabular}{|c|c|c|c|c|c|c|c|c|c|c|c|c|c|c|c|}
\hline \multirow[b]{2}{*}{ Characteristics } & \multicolumn{5}{|c|}{$2-8$ years $(n 128)$} & \multicolumn{5}{|c|}{$9-18$ years $(n 226)$} & \multicolumn{5}{|c|}{$\geq 19$ years $(n 731)$} \\
\hline & $\begin{array}{c}\text { Total } \\
\text { Canadian } \\
\text { population }(\%) \\
\end{array}$ & OR & $95 \% \mathrm{Cl}$ & Mean & SE & $\begin{array}{c}\text { Total } \\
\text { Canadian } \\
\text { population (\%) }\end{array}$ & OR & $95 \% \mathrm{Cl}$ & Mean & SE & $\begin{array}{c}\text { Total } \\
\text { Canadian } \\
\text { population (\%) } \\
\end{array}$ & OR & $95 \% \mathrm{Cl}$ & Mean & SE \\
\hline \multicolumn{16}{|l|}{ Sex } \\
\hline Male & $2 \cdot 2$ & Reference & & $12 \cdot 0$ & 4.2 & 3.1 & Reference & & 7.5 & 1.6 & 4.3 & Reference & & 14.0 & 3.5 \\
\hline Female & 3.6 & 1.68 & $0.72,3.96$ & $7 \cdot 0$ & 1.9 & 3.7 & 1.20 & $0.71,2 \cdot 02$ & $7 \cdot 1$ & 1.7 & 5.9 & 1.40 & $1.02,1.91$ & $9 \cdot 0$ & $1 \cdot 2$ \\
\hline \multicolumn{16}{|l|}{ Provincial location } \\
\hline Maritimes & 1.6 & Reference & & 7.0 & $5 \cdot 7$ & 1.2 & Reference & & 3.0 & 1.3 & 9.8 & Reference & & 13.0 & 4.8 \\
\hline Quebec & 1.6 & 0.98 & $0.18,5.46$ & 7.0 & 1.6 & 2.7 & 2.38 & $0 \cdot 78,7 \cdot 20$ & 5.0 & 1.6 & 3.7 & 1.33 & $0.63,2.79$ & 8.0 & $2 \cdot 7$ \\
\hline Ontario & 3.1 & 1.97 & $0.52,7.41$ & $5 \cdot 0$ & 2.7 & 3.7 & 3.26 & $1.35,7.83$ & 8.0 & 1.8 & $5 \cdot 3$ & 1.96 & $0.58,6.63$ & 11.0 & 3.0 \\
\hline Prairies & 4.0 & 2.0 & $0.31,5 \cdot 30$ & 21.0 & $12 \cdot 0$ & 1.9 & 1.64 & $0.62,4.36$ & 7.0 & 2.7 & 10.0 & 1.48 & $0.80,2.74$ & 9.0 & $2 \cdot 3$ \\
\hline British Columbia & 6.6 & 4.34 & $1.07,17.57$ & 11.0 & 3.8 & 7.4 & 6.86 & $1 \cdot 87,25 \cdot 18$ & 8.0 & 2.0 & $10 \cdot 2$ & 4.00 & $1.5,10.68$ & 13.0 & $2 \cdot 9$ \\
\hline Overall & 3.4 & & & 9.0 & $2 \cdot 2$ & 3.4 & & & $7 \cdot 0$ & 0.9 & $5 \cdot 2$ & & & $10 \cdot 8$ & 6.5 \\
\hline \multicolumn{16}{|l|}{ Cultural background } \\
\hline $\begin{array}{l}\text { African, Latin, } \\
\text { Arabic, Aboriginal } \\
\text { or other }\end{array}$ & 1.4 & Reference & & $27 \cdot 0$ & 35.5 & $2 \cdot 2$ & Reference & & 5.0 & 5.09 & 1.4 & Reference & & $10 \cdot 0$ & $2 \cdot 1$ \\
\hline Caucasian & $2 \cdot 3$ & 0.58 & $0.03,10.27$ & $10 \cdot 0$ & 2.7 & $2 \cdot 7$ & 0.97 & $0.28,3.39$ & 6.0 & 0.9 & 3.0 & 1.03 & $0.30,3.59$ & $7 \cdot 0$ & $6 \cdot 0$ \\
\hline $\begin{array}{l}\text { Asian Canadian } \\
\text { Income* }\end{array}$ & 6.9 & 3.15 & $1.03,9.66$ & 5.0 & 1.8 & 11 & 4.26 & $2.39,7.58$ & $10 \cdot 0$ & 2.0 & 8.7 & 4.38 & $1.56,12 \cdot 35$ & $13 \cdot 0$ & 1.7 \\
\hline $\begin{array}{l}\text { Income }^{*} \\
\text { Lowest }\end{array}$ & \multicolumn{7}{|c|}{ Income ${ }^{*}$} & & & & 4.7 & Reference & & $7 \cdot 0$ & 3.9 \\
\hline Lower middle & & & & & & & & & & & 5.5 & 1.18 & $0.50,2.79$ & $12 \cdot 0$ & $5 \cdot 0$ \\
\hline Upper middle & & & & & & & & & & & 4.0 & 0.91 & $0.47,1.78$ & $12 \cdot 0$ & 3.0 \\
\hline Highest & & & & & & & & & & & 6.0 & 0.98 & $0.47,2.03$ & 9.0 & 2.0 \\
\hline \multicolumn{16}{|l|}{ Education* } \\
\hline$<$ Secondary school & & NA & & & & & NA & & & & 2.5 & Reference & & $10 \cdot 0$ & 3.4 \\
\hline Secondary school & & & & & & & & & & & 4.7 & 1.94 & $0.71,5.34$ & $10 \cdot 0$ & $2 \cdot 8$ \\
\hline Post-secondary school & & & & & & & & & & & $5 \cdot 1$ & $2 \cdot 12$ & $1.02,4.44$ & 13.0 & 7.1 \\
\hline $\begin{array}{l}\text { Post-secondary } \\
\text { degree/diploma }\end{array}$ & & & & & & & & & & & 6.3 & 2.68 & $1.58,4.57$ & $11 \cdot 0$ & $2 \cdot 1$ \\
\hline
\end{tabular}

NA, not determined.

* Income and education were not examined among the respondents aged $<19$ years. 


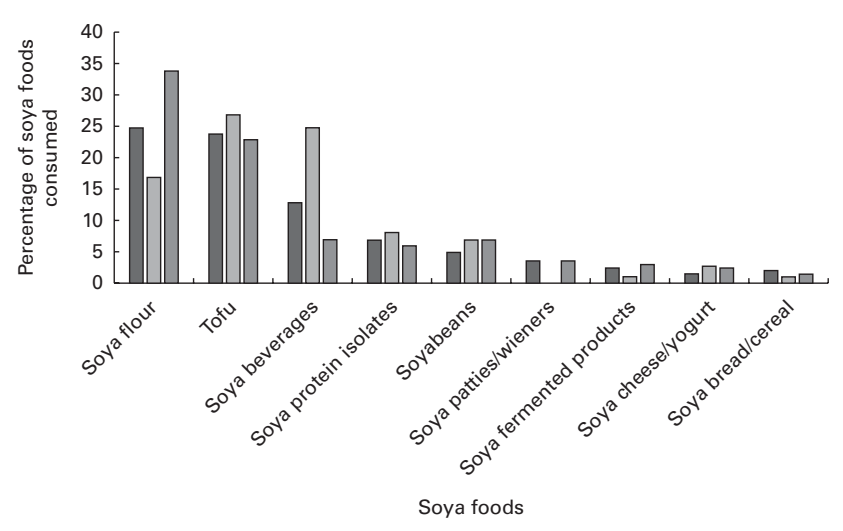

Fig. 1. Most commonly consumed food sources of soya products by age group in the Canadian diet $(1 \mathrm{~d}, 24 \mathrm{~h}$ dietary recall of the Canadian Community Health Survey, Cycle 2.2 (2004)). $\square, 2-8$ years; $\square, 9-18$ years; $\square, \geq 19$ years.

folate (33\%), pteroyl(mono)glutamic acid (32\%), Ca (53\%), $\mathrm{P} 14 \%), \mathrm{Mg}(32 \%), \mathrm{Fe}(35 \%)$ and $\mathrm{K}(15 \%)$. When total energy intake was accounted for, intakes were significantly higher for thiamin, vitamin $\mathrm{B}_{6}, \mathrm{Ca}, \mathrm{P}, \mathrm{Mg}$ and $\mathrm{Fe}$ in high soya consumers than those in non-consumers, as well as lower for saturated fat $(-20 \%)$. No major differences were found comparing adult soya consumers in the low-soya consumption group (who consumed on average $1.5 \mathrm{~g}$ soya protein) with non-consumers, with the exception of higher fibre $/ 4184 \mathrm{~kJ}$ and lower intakes of vitamin D/4184 kJ (Table 4). In terms of food group intake, adult consumers in both soya groups consumed approximately 1 serving greater of fruits and vegetables than their non-consumer counterparts. Respondents with high soya intakes consumed 1.5 servings more of meat and alternatives than both non-consumers and low soya consumers (Table 4).

\section{Discussion}

On any given day, $3.3 \%$ of Canadians consume soya products. This is similar to US data from two $24 \mathrm{~h}$ recalls on soya consumption patterns in respondents aged 9-70 years, in which case there were a total of 226 soya products mentioned by 5510 individuals $^{(22)}$. If it is assumed that each mention was made by separate respondents, the consumption rate using the data from the National Health and Nutrition Examination Survey (NHANES) would be $4 \cdot 1 \%$; this is similar to the results of the CCHS 2.2 using both 1 and $2 \mathrm{~d}$ recalls ( $4.2 \%$, data not shown). In a $1 \mathrm{~d}$ recall interview in the EPIC study of the European population, only $1.9 \%$ of respondents consumed soya. However, results from the EPIC study based on sex differences were similar to those in the CCHS 2.2 analysis, with females being more likely to be soya consumers than males $^{(23)}$. The sex-based difference in consumption patterns may be due to adult females consuming soya products for their hypothesised bone-protective effects ${ }^{(2,14,15)}$.

Differences between the European and Canadian consumption rates may be attributed to Canada's large Asian population, which is of the order of $10 \%$, making up approximately $66 \%$ of Canada's visible minority population ${ }^{(35)}$, while Asians represent a much smaller proportion of the European population $^{(36)}$. Indeed, Asian Canadian adults and children were significantly more likely to be soya consumers than any other cultural group, and the province of British Columbia has a high Asian population ${ }^{(37)}$.

Previous studies have suggested that socio-economic status may influence soya consumption patterns ${ }^{(38-40)}$. The present study found that individuals with a higher level of education were more likely to consume soya, consistent with previous research which has shown that the nutrition and health perceptions of soya foods increase with education level ${ }^{(41)}$ and knowledge of soya as a functional food ${ }^{(42)}$. Similarly, lack of knowledge (preparation techniques and associated health benefits) has been cited as a hindrance to soya consumption $^{(43)}$. Although income level did not influence the consumption of soya products in the present study, research has indicated that the cost of soya foods is a major barrier to soya consumption, particularly among low-income adults ${ }^{(39)}$.

Western soya consumption differs greatly from Asian soya consumption that is higher in 'traditional' soya foods such as tofu, tempeh, miso and natto (boiled and fermented soyabeans) ${ }^{(24)}$. The types of soya foods consumed also differed in this cohort when compared with US and European studies. Soya flour (all forms) accounted for more than one-third of the soya products consumed by adults in the CCHS 2.2, while in Europe, it was the third most reported item (grouped with other soya grain products), and did not even appear in the results of the NHANES. In the US population, the most common form of soya consumed was soya sauce (which was excluded in the present study), followed by meat replacement products (which were not highly consumed among Canadians, regardless of age group), soya replacement drinks/bars, soya milk and tofu. Tofu consumption was also more prevalent in Canada than in the USA. Comparably, Western European consumers ate more soya dairy substitutes (milk, cream, drinks, cheese and yogurt) ${ }^{(22,23)}$.

Canadian soya protein intake averaged between 7 and $14 \mathrm{~g} / \mathrm{d}$ depending on age and sex. Messina and colleagues ${ }^{(24)}$ observed soya protein consumption in adults in four Asian countries, and found that soya protein intake varied depending on age, sex and region. Older Japanese adults consumed between 6 and $11 \mathrm{~g}$ soya protein, while residents of Shanghai had a daily mean soya protein intake of $8.8 \mathrm{~g}$. Conversely, Singapore data show lower soya protein intake $(\leq 5 \cdot 1 \mathrm{~g} / \mathrm{d})$ among residents.

The present analyses show that Canadians who consume soya products have higher nutrient intakes than nonconsumers. Previous simulation analyses predicted that dietary intakes of some nutrients would be increased, while others would be compromised if soya foods replaced milk and meats in the diet ${ }^{(22)}$. Many soya products are good sources of carbohydrates and soya protein is a high-quality protein source ${ }^{(2)}$, which was evidenced in the higher intakes of these macronutrients by adult soya consumers. In their simulation, Tucker et $a l^{(22)}$ predicted that replacing milk with a soya dairy beverage would result in greater $\mathrm{Mg}, \mathrm{Fe}$ and fibre intakes, all of which were observed in the present study, probably due to the fact that soya is rich in these nutrients. Thiamin, which was also found to be significantly higher 
Table 2. Soya protein amount and nutrient intakes per d for soya consumers and non-consumers aged 2-8 years based on $1 \mathrm{~d}$ intakes from the Canadian Community Health Survey, Cycle 2.22004

(Mean values with their standard errors; $n$ 4105)

\begin{tabular}{|c|c|c|c|c|c|c|}
\hline \multirow[b]{2}{*}{ Intake categories } & \multicolumn{2}{|c|}{$\begin{array}{c}\text { Non-consumers } \\
\quad(n \text { 3977) }\end{array}$} & \multicolumn{2}{|c|}{$\begin{array}{l}\text { Low consumers } \\
\quad(n 64)\end{array}$} & \multicolumn{2}{|c|}{$\begin{array}{l}\text { High consumers } \\
(n \text { 64) }\end{array}$} \\
\hline & Mean & SE & Mean & $\mathrm{SE}$ & Mean & $\mathrm{SE}$ \\
\hline Soya protein intake $(\mathrm{g})$ & \multicolumn{2}{|c|}{0} & 1.5 & 0.5 & $16 \cdot 6$ & $4 \cdot 1$ \\
\hline Food amount (g) & 1922 & 26 & 1846 & 140 & 1964 & 131 \\
\hline \multicolumn{7}{|l|}{ Energy } \\
\hline $\mathrm{kcal}$ & 1777 & 37 & 1598 & 173 & 1679 & 103 \\
\hline $\mathrm{kJ}$ & 7435 & 154 & 6686 & 724 & 7025 & 431 \\
\hline Protein $(\mathrm{g})$ & 65 & 1.4 & 59 & $7 \cdot 8$ & 71 & $5 \cdot 1$ \\
\hline Protein $(\mathrm{g} / 4184 \mathrm{~kJ})$ & 37 & 0.5 & 37 & $3 \cdot 3$ & $43^{*}$ & 3 \\
\hline Carbohydrate $(\mathrm{g})$ & 245 & 5 & 231 & 33 & 233 & 20 \\
\hline Carbohydrate $(\mathrm{g} / 4184 \mathrm{~kJ})$ & 141 & 1 & 145 & 10 & 139 & 6 \\
\hline Fibre $(\mathrm{g})$ & $12 \cdot 4$ & 0.3 & $15 \cdot 5$ & 4.7 & 14.2 & 1.3 \\
\hline Fibre $(\mathrm{g} / 4184 \mathrm{~kJ})$ & 7 & 0.1 & 10 & $2 \cdot 3$ & $9^{\star}$ & 0.7 \\
\hline Sugar (g) & 117 & 2 & $86^{\star \star}$ & 11 & 104 & 13 \\
\hline Total fat $(\mathrm{g})$ & 62 & 1.6 & 51 & 7 & 55 & $6 \cdot 2$ \\
\hline Total fat $(\mathrm{g} / 4184 \mathrm{~kJ})$ & 34 & 0.5 & 32 & $2 \cdot 6$ & 32 & $2 \cdot 3$ \\
\hline SFA (g) & 23 & 0.6 & $18^{\star \star}$ & $1 \cdot 8$ & $19^{\star \star}$ & 1.5 \\
\hline SFA (g/4184 kJ) & 10 & 0.3 & 10 & 0.7 & 9 & 0.6 \\
\hline MUFA (g) & 23 & 0.8 & 19 & $3 \cdot 1$ & 19 & $3 \cdot 1$ \\
\hline MUFA $(\mathrm{g} / 4184 \mathrm{~kJ})$ & 7.0 & 0.2 & $7 \cdot 2$ & $1 \cdot 1$ & $6 \cdot 1$ & 0.7 \\
\hline PUFA (g) & 9 & 0.3 & 9 & 1.7 & 11 & $2 \cdot 1$ \\
\hline PUFA $(\mathrm{g} / 4184 \mathrm{~kJ})$ & $2 \cdot 8$ & 0.07 & $3 \cdot 2$ & 0.4 & 3.3 & 0.3 \\
\hline Linoleic acid $(\mathrm{g})$ & $7 \cdot 6$ & 0.3 & 7.4 & $1 \cdot 7$ & 9.4 & 1.7 \\
\hline Linoleic acid $(\mathrm{g} / 4184 \mathrm{~kJ})$ & 4.2 & 0.1 & 4.5 & 0.8 & $5 \cdot 3$ & 0.6 \\
\hline Linolenic acid $(\mathrm{g})$ & 1.2 & 0.05 & 1.1 & 0.2 & 1.3 & 0.3 \\
\hline Linolenic acid (g/4184 kJ) & $0 . \overline{7}$ & 0.02 & $0 \cdot 7$ & $0 \cdot 1$ & \multirow{2}{*}{\multicolumn{2}{|c|}{$7 \cdot 2: 1$}} \\
\hline Linoleic:linolenic ratio & \multicolumn{2}{|c|}{$6 \cdot 3: 1$} & \multicolumn{2}{|c|}{$6 \cdot 7: 1$} & & \\
\hline Cholesterol (mg) & 201 & 7.6 & 175 & 33.2 & 169 & 28.5 \\
\hline Cholesterol (mg/4184 kJ) & 113 & $2 \cdot 7$ & 111 & 22.4 & 107 & $22 \cdot 4$ \\
\hline Vitamin A $(\mu \mathrm{g})$ & 576 & 13 & $471 \dagger$ & 55 & 627 & 132 \\
\hline Vitamin A $(\mu \mathrm{g} / 4184 \mathrm{~kJ})$ & 333 & $7 \cdot 2$ & 301 & 53 & 387 & 68.5 \\
\hline Vitamin C (mg) & 145 & 4 & 122 & 23 & 170 & 461 \\
\hline Vitamin C (mg/4184kJ) & 84 & 2.9 & 81 & 15 & 101 & 20 \\
\hline Thiamin $(\mathrm{mg})$ & 1.5 & 0.03 & 1.4 & 0.3 & 1.6 & 0.13 \\
\hline Thiamin (mg/4184 kJ) & 0.9 & 0.01 & 0.9 & 0.1 & $1.0^{*}$ & 0.06 \\
\hline Riboflavin (mg) & $2 \cdot 0$ & 0.05 & $1 \cdot 7$ & 0.2 & 1.8 & 0.2 \\
\hline Riboflavin $(\mathrm{mg} / 4184 \mathrm{~kJ})$ & 1.1 & 0.03 & 1.0 & 0.1 & 1.1 & 0.09 \\
\hline Niacin $(\mathrm{mg})$ & $27 \cdot 7$ & 0.6 & $25 \cdot 8$ & $4 \cdot 1$ & 29.8 & $2 \cdot 1$ \\
\hline Niacin (mg/4184 kJ) & $15 \cdot 7$ & 0.2 & 16 & 1.3 & $18 \dagger$ & $1 \cdot 2$ \\
\hline Vitamin $B_{6}(\mathrm{mg})$ & 1.4 & 0.02 & 1.3 & 0.18 & 1.5 & 0.1 \\
\hline Vitamin $B_{6}(\mathrm{mg} / 4184 \mathrm{~kJ})$ & 0.8 & 0.02 & 0.8 & 0.05 & $0.9 \dagger$ & 0.05 \\
\hline Vitamin $B_{12}(\mu \mathrm{g})$ & 3.5 & $0 \cdot 1$ & 3.0 & 0.4 & 3.3 & 0.3 \\
\hline Vitamin $B_{12}(\mu \mathrm{g} / 4184 \mathrm{~kJ})$ & $2 \cdot 0$ & 0.07 & 1.9 & 0.3 & $2 \cdot 1$ & 0.3 \\
\hline Naturally occurring folate $(\mu \mathrm{g})$ & 163 & $4 \cdot 8$ & 199 & 50 & 193 & 19 \\
\hline Folic acid $(\mu \mathrm{g})$ & 110 & 3.4 & 107 & 20.4 & 97 & $21 \cdot 6$ \\
\hline Folate (from food in dietary folate equivalents) ( $\mu \mathrm{g}$ ) & 363 & 14 & 351 & 78 & 374 & 41 \\
\hline Folate $(\mu \mathrm{g} / 4184 \mathrm{~kJ})$ & 205 & 3.9 & 216 & $32 \cdot 8$ & 219 & $16 \cdot 5$ \\
\hline Vitamin D $(\mu \mathrm{g})$ & 6.0 & 0.2 & $6 \cdot 2$ & 0.8 & $6 \cdot 0$ & 0.7 \\
\hline Vitamin $D(\mu \mathrm{g} / 4184 \mathrm{~kJ})$ & 3.5 & $0 \cdot 1$ & 3.9 & 0.8 & 3.7 & 0.5 \\
\hline $\mathrm{Ca}(\mathrm{mg})$ & 1028 & 20.5 & 1019 & 158 & 1213 & 136 \\
\hline $\mathrm{Ca}(\mathrm{mg} / 4184 \mathrm{~kJ})$ & 594 & 16 & 651 & 82 & $731^{*}$ & $64^{\star}$ \\
\hline$P(\mathrm{mg})$ & 1209 & $19 \cdot 8$ & 1122 & 124.6 & 1183 & $79 \cdot 1$ \\
\hline $\mathrm{P}(\mathrm{mg} / 4184 \mathrm{~kJ})$ & 692 & 11 & 706 & 53 & 726 & 55 \\
\hline $\mathrm{Mg}(\mathrm{mg})$ & 245 & 3.6 & 238 & $27 \cdot 7$ & 273 & $19 \cdot 3$ \\
\hline $\mathrm{Mg}(\mathrm{mg} / 4184 \mathrm{~kJ})$ & 141 & $2 \cdot 7$ & 151 & 8.2 & $166^{\star \star}$ & $9 \cdot 3^{* *}$ \\
\hline $\mathrm{Fe}(\mathrm{mg})$ & 12 & 0.3 & 10 & 1.2 & 14 & 1.5 \\
\hline $\mathrm{Fe}(\mathrm{mg} / 4184 \mathrm{~kJ})$ & $6 \cdot 7$ & 0.1 & $6 \cdot 3$ & 0.4 & $8.1^{*}$ & 0.6 \\
\hline $\mathrm{Zn}(\mathrm{mg})$ & 9 & 0.2 & 8 & 0.9 & 9 & 0.7 \\
\hline $\mathrm{Zn}(\mathrm{mg} / 4184 \mathrm{~kJ})$ & 4.9 & 0.06 & $5 \cdot 0$ & 0.3 & 5.5 & 0.4 \\
\hline $\mathrm{Na}(\mathrm{mg})$ & 2480 & 78 & 1991 & 276 & 2377 & 205 \\
\hline $\mathrm{Na}(\mathrm{mg} / 4184 \mathrm{~kJ})$ & 1396 & 21 & $1241 t$ & 78 & 1439 & 42 \\
\hline $\mathrm{K}(\mathrm{mg})$ & 2523 & 38 & 2333 & 211 & 2607 & 166 \\
\hline $\mathrm{K}(\mathrm{mg} / 4184 \mathrm{~kJ})$ & 1455 & $26 \cdot 1$ & 1477 & 86 & 1617 & 118 \\
\hline Grain products (servings) & $5 \cdot 2$ & 0.1 & 5.6 & 1.1 & 4.8 & 0.7 \\
\hline Fruit and vegetable products (servings) & $4 \cdot \overline{3}$ & 0.1 & 4.0 & 0.7 & $5 \cdot 0$ & 1.0 \\
\hline Milk and milk products (servings) & 2.4 & 0.06 & 1.9 & 0.5 & $2 \cdot 2$ & 0.3 \\
\hline Meat and alternatives (servings) & $2 \cdot 3$ & 0.1 & 2.5 & 0.5 & 3.5 & 0.5 \\
\hline
\end{tabular}

Mean value was significantly different from that of non-consumers: ${ }^{\star} P<0.05,{ }^{\star \star} P<0.01,{ }^{\star \star \star} P<0.001$.

$\dagger$ Mean value was different from that of non-consumers $(0.1<P<0.05$; trend). 
Table 3. Soya protein amount and nutrient intakes per $d$ for soya consumers and non-consumers aged 9-18 years based on $1 \mathrm{~d}$ intakes from the Canadian Community Health Survey, Cycle 2.22004

(Mean values with their standard errors; $n$ 8957)

\begin{tabular}{|c|c|c|c|c|c|c|}
\hline \multirow[b]{2}{*}{ Intake categories } & \multicolumn{2}{|c|}{$\begin{array}{l}\text { Non-consumers } \\
\quad(n \text { 8731) }\end{array}$} & \multicolumn{2}{|c|}{$\begin{array}{l}\text { Low consumers } \\
\qquad(n 113)\end{array}$} & \multicolumn{2}{|c|}{$\begin{array}{l}\text { High consumers } \\
\qquad(n 113)\end{array}$} \\
\hline & Mean & SE & Mean & SE & Mean & SE \\
\hline Soya protein intake (g) & \multicolumn{2}{|c|}{0} & 0.8 & 0.12 & $12 \cdot 3$ & 1.5 \\
\hline Food amount (g) & 2701 & 34 & 2830 & 284 & $3288^{*}$ & $223^{*}$ \\
\hline \multicolumn{7}{|l|}{ Energy } \\
\hline $\mathrm{kcal}$ & 2360 & 41 & 2332 & 265 & 2662 & 203 \\
\hline $\mathrm{kJ}$ & 9874 & 172 & 9757 & 1109 & 11138 & 849 \\
\hline Protein $(\mathrm{g})$ & 86 & 1.5 & 87 & 8.7 & $109^{\star}$ & $8 \cdot 5^{\star}$ \\
\hline Protein (g/4184 kJ) & 37 & 0.35 & 38 & $2 \cdot 1$ & $41 \dagger$ & $2 \cdot 6$ \\
\hline Carbohydrate $(\mathrm{g})$ & 320 & $5 \cdot 7$ & 309 & $39 \cdot 0$ & 366 & 33.4 \\
\hline Carbohydrate (g/4184 kJ) & 137 & 0.5 & 135 & 4.3 & 139 & 4.0 \\
\hline Fibre $(g)$ & $15 \cdot 7$ & 0.2 & 17.4 & $2 \cdot 1$ & $19 \cdot 7^{\star}$ & 1.5 \\
\hline Fibre $(\mathrm{g} / 4184 \mathrm{~kJ})$ & 6.9 & 0.08 & $8 \cdot 1$ & 0.91 & $7.9 \dagger$ & 0.62 \\
\hline Sugar $(g)$ & 146 & 3.0 & 136 & $20 \cdot 2$ & 157 & 11.4 \\
\hline Total fat (g) & 84 & 1.4 & 86 & $10 \cdot 6$ & 88 & $7 \cdot 1$ \\
\hline Total fat $(\mathrm{g} / 4184 \mathrm{~kJ})$ & 35 & 0.19 & 36 & 1.80 & 33 & 1.30 \\
\hline SFA (g) & 29 & 0.6 & 29 & 3.5 & 29 & $2 \cdot 6$ \\
\hline Saturated fat (g/4184 kJ) & 14 & 0.7 & 12 & 0.3 & 10 & 0.4 \\
\hline MUFA (g) & 33 & 0.5 & 34 & 4.8 & 34 & $2 \cdot 8$ \\
\hline MUFA $(\mathrm{g} / 4184 \mathrm{~kJ})$ & $6 \cdot 1$ & 0.1 & $6 \cdot 2$ & 0.6 & $4 \cdot 7^{\star \star \star}$ & 0.3 \\
\hline PUFA (g) & 14 & 0.28 & 14 & $2 \cdot 2$ & 17 & $1 \cdot 8$ \\
\hline PUFA (g/4184 kJ) & $3 \cdot 6$ & 0.06 & $2 \cdot 6$ & 0.3 & $2 \cdot 4$ & 0.2 \\
\hline Linoleic acid $(\mathrm{g})$ & 11 & 0.3 & 12 & 1.9 & $14 \dagger$ & 1.4 \\
\hline Linoleic acid (g/4184 kJ) & 4.8 & 0.06 & $5 \cdot 1$ & 0.50 & $5 \cdot 2$ & 0.30 \\
\hline Linolenic acid (g) & $1 \cdot 8$ & $1 \cdot 7$ & 1.9 & 0.4 & $2 \cdot 1$ & 0.2 \\
\hline Linolenic acid (g/4184 kJ) & 0.8 & 0.01 & 0.76 & 0.12 & 0.79 & 0.06 \\
\hline Linoleic:linolenic ratio & \multicolumn{2}{|c|}{$6 \cdot 1: 1$} & \multicolumn{2}{|c|}{$6 \cdot 3: 1$} & \multicolumn{2}{|c|}{$6 \cdot 7: 1$} \\
\hline Cholesterol (mg) & 255 & $6 \cdot 4$ & 282 & 53.2 & $330 \dagger$ & $39 \cdot 4$ \\
\hline Cholesterol (mg/4184kJ) & 108 & 1.6 & 120 & $19 \cdot \overline{6}$ & 123 & $15 \cdot 6$ \\
\hline Vitamin A $(\mu \mathrm{g})$ & 652 & 13 & 756 & 146 & 783 & 104 \\
\hline Vitamin A $(\mu \mathrm{g} / 4184 \mathrm{~kJ})$ & 286 & $5 \cdot 0$ & 354 & $75 \cdot 3$ & 312 & 44.7 \\
\hline Vitamin C (mg) & 152 & $3 \cdot 6$ & 175 & $41 \cdot 6$ & $221 \dagger$ & $34 \cdot 1$ \\
\hline Vitamin C (mg/4184 kJ) & 68 & 1.4 & 83 & $23 \cdot 3$ & 89 & $14 \cdot 1$ \\
\hline Thiamin $(\mathrm{mg})$ & 1.9 & 0.06 & 1.9 & 0.21 & $2 \cdot 3 \dagger$ & 0.2 \\
\hline Thiamin (mg/4184 kJ) & 0.83 & 0.01 & 0.81 & 0.05 & 0.88 & 0.05 \\
\hline Riboflavin (mg) & $2 \cdot 2$ & 0.05 & $2 \cdot 2$ & 0.28 & 2.5 & 0.19 \\
\hline Riboflavin (mg/4184 kJ) & 0.97 & 0.01 & 0.95 & 0.12 & 0.97 & 0.07 \\
\hline Niacin $(\mathrm{mg})$ & 38.4 & 0.7 & 37.8 & 3.8 & $45.0 \dagger$ & 3.4 \\
\hline Niacin (mg/4184 kJ) & $16 \cdot 5$ & 0.17 & $16 \cdot 4$ & 0.83 & $17 \cdot 1$ & 0.92 \\
\hline Vitamin $B_{6}(\mathrm{mg})$ & 1.8 & 0.03 & 1.7 & 0.18 & $2 \cdot 4^{\star \star}$ & 0.21 \\
\hline Vitamin $B_{6}(\mathrm{mg} / 4184 \mathrm{~kJ})$ & 0.8 & 0.01 & 0.8 & 0.06 & $1.0^{*}$ & 0.09 \\
\hline Vitamin $B_{12}(\mu \mathrm{g})$ & 4.2 & 0.12 & 4.0 & 0.57 & $6 \cdot 2$ & $2 \cdot 13$ \\
\hline Vitamin $B_{12}(\mu \mathrm{g} / 4184 \mathrm{~kJ})$ & $1 \cdot 8$ & 0.03 & $1 \cdot 8$ & 0.19 & $2 \cdot 1$ & 3.4 \\
\hline Naturally occurring folate $(\mu \mathrm{g})$ & 205 & 4.4 & 248 & 34.0 & $295^{\star \star \star}$ & $23 \cdot 3$ \\
\hline Folic acid $(\mu \mathrm{g})$ & 146 & 2.5 & 148 & 24.9 & 295 & $23 \cdot 2$ \\
\hline Folate (from food in dietary folate equivalents) $(\mu \mathrm{g})$ & 480 & 11 & 495 & 54 & 544 & 40 \\
\hline Folate $(\mu \mathrm{g} / 4184 \mathrm{~kJ})$ & 208 & $2 \cdot 2$ & 219 & $22 \cdot 2$ & 211 & $12 \cdot 3$ \\
\hline Vitamin $D(\mu g)$ & $6 \cdot 3$ & 0.13 & 6.5 & 1.87 & $7 \cdot 1$ & 0.93 \\
\hline Vitamin D $(\mu \mathrm{g} / 4184 \mathrm{~kJ})$ & $2 \cdot 7$ & 0.04 & 2.8 & 0.74 & 2.6 & 0.26 \\
\hline $\mathrm{Ca}(\mathrm{mg})$ & 1097 & 17 & 1085 & 181 & $1472^{\star *}$ & 139 \\
\hline $\mathrm{Ca}(\mathrm{mg} / 4184 \mathrm{~kJ})$ & 474 & 5.4 & 480 & $70 \cdot 7$ & $568^{\star}$ & $42 \cdot 3$ \\
\hline $\mathrm{P}(\mathrm{mg})$ & 1450 & 23 & 1476 & 172 & $1763^{*}$ & 125 \\
\hline$P(\mathrm{mg} / 4184 \mathrm{~kJ})$ & 621 & 4.8 & 647 & 47 & 673 & 45 \\
\hline $\mathrm{Mg}(\mathrm{mg})$ & 301 & $3 \cdot 8$ & 318 & $39 \cdot 2$ & $395^{\star \star \star}$ & $24 \cdot 8$ \\
\hline $\mathrm{Mg}(\mathrm{mg} / 4184 \mathrm{~kJ})$ & 131 & 1.4 & 145 & $15 \cdot 5$ & $157^{\star \star}$ & $10 \cdot 3$ \\
\hline $\mathrm{Fe}(\mathrm{mg})$ & $15 \cdot 5$ & 0.38 & 14.9 & 1.3 & $19 \cdot 3^{*}$ & 1.7 \\
\hline $\mathrm{Fe}(\mathrm{mg} / 4184 \mathrm{~kJ})$ & $6 \cdot 7$ & 0.06 & $6 \cdot 6$ & 0.50 & $7 \cdot 4$ & 0.05 \\
\hline $\mathrm{Zn}(\mathrm{mg})$ & 11.5 & 0.2 & $12 \cdot 1$ & 1.3 & $14 \cdot 4^{*}$ & 1.3 \\
\hline $\mathrm{Zn}(\mathrm{mg} / 4184 \mathrm{~kJ})$ & 4.9 & 0.05 & $5 \cdot 3$ & 0.40 & 5.5 & 0.40 \\
\hline $\mathrm{Na}(\mathrm{mg})$ & 3387 & 64 & 3700 & 632 & 3650 & 368 \\
\hline $\mathrm{Na}(\mathrm{mg} / 4184 \mathrm{~kJ})$ & 1458 & $12 \cdot 2$ & 1574 & 92.7 & 1390 & $115 \cdot 0$ \\
\hline $\mathrm{K}(\mathrm{mg})$ & 3017 & 46 & 3116 & 358 & $3771^{\star}$ & 293 \\
\hline $\mathrm{K}(\mathrm{mg} / 4184 \mathrm{~kJ})$ & 1312 & $13 \cdot 2$ & 1394 & $105 \cdot 2$ & 1474 & $107 \cdot 0$ \\
\hline Grain products (servings) & $6 \cdot 7$ & 0.14 & $6 \cdot 3$ & 0.7 & $7 \cdot 6$ & 0.7 \\
\hline Fruit and vegetable products (servings) & 4.7 & 0.09 & 4.9 & 0.5 & $7 \cdot 5^{\star \star}$ & 1.05 \\
\hline Milk and milk products (servings) & $2 \cdot 4$ & 0.04 & $2 \cdot 4$ & 0.05 & 2.5 & 0.3 \\
\hline Meat and alternatives (servings) & 3.5 & 0.07 & 3.6 & 0.5 & $4 \cdot 8^{\star \star}$ & 0.5 \\
\hline
\end{tabular}

Mean value was significantly different from that of non-consumers: ${ }^{*} P<0.05,{ }^{* \star} P<0.01,{ }^{* \star *} P<0.001$.

$\dagger$ Mean value was different from that of non-consumers $(0.1<P<0.05$; trend). 
Table 4. Soya protein amount and nutrient intakes per $d$ for non-consumers and soya consumers aged $\geq 19$ years based on $1 \mathrm{~d}$ intakes from the Canadian Community Health Survey, Cycle 2.22004

(Mean values with their standard errors; $n 20156$ )

\begin{tabular}{|c|c|c|c|c|c|c|}
\hline \multirow[b]{2}{*}{ Intake categories } & \multicolumn{2}{|c|}{$\begin{array}{l}\text { Non-consumers } \\
\quad(n \text { 19 425) }\end{array}$} & \multicolumn{2}{|c|}{$\begin{array}{l}\text { Low consumers } \\
\qquad(n 365)\end{array}$} & \multicolumn{2}{|c|}{$\begin{array}{l}\text { High consumers } \\
\qquad(n 366)\end{array}$} \\
\hline & Mean & SE & Mean & SE & Mean & SE \\
\hline Soya protein intake (g) & \multicolumn{2}{|c|}{0} & 1.5 & 0.12 & $16 \cdot 5$ & $2 \cdot 0$ \\
\hline Food amount (g) & 3232 & 130 & 3485 & 264 & $3563^{\star \star}$ & 193 \\
\hline \multicolumn{7}{|l|}{ Energy } \\
\hline $\mathrm{kcal}$ & 2088 & 183 & 1993 & 125 & 2298 & 260 \\
\hline $\mathrm{kJ}$ & 8736 & 766 & 8339 & 523 & 9615 & 1088 \\
\hline Protein $(\mathrm{g})$ & 85 & 7 & 72 & 8 & $108^{\star}$ & 19 \\
\hline Protein (g/4184 kJ) & $41 \cdot 8$ & 0.3 & $42 \cdot 1$ & $2 \cdot 7$ & $47 \cdot 2 \dagger$ & $3 \cdot 1$ \\
\hline Carbohydrate $(\mathrm{g})$ & 256 & 21 & 256 & 13 & $279^{\star}$ & 24 \\
\hline Carbohydrate (g/4184 kJ) & 125 & $1 \cdot 3$ & $131 \dagger$ & 2.9 & 125 & $4 \cdot 3$ \\
\hline Fibre $(g)$ & $17 \cdot 1$ & $1 \cdot 2$ & $18 \cdot 9$ & 0.9 & $23 \cdot 3^{\star \star \star}$ & $1 \cdot 1$ \\
\hline Fibre $(\mathrm{g} / 4184 \mathrm{~kJ})$ & $8 \cdot 7$ & 0.2 & $10 \cdot 0^{\star \star \star}$ & 0.4 & 10.5 & 1.4 \\
\hline Sugar $(g)$ & 104 & 7 & 94 & 12 & 101 & 7 \\
\hline Total fat $(\mathrm{g})$ & $76 \cdot 3$ & 7.5 & $67 \cdot 3$ & $7 \cdot 1$ & $80 \cdot 2$ & $9 \cdot 9$ \\
\hline Total fat $(\mathrm{g} / 4184 \mathrm{~kJ})$ & 36 & 0.47 & 32 & $2 \cdot 13$ & 34 & 1.02 \\
\hline SFA $(g)$ & 24.9 & $2 \cdot 8$ & $22 \cdot 0$ & $3 \cdot 3$ & $23 \cdot 0$ & 4.4 \\
\hline Saturated fat $(\mathrm{g} / 4184 \mathrm{~kJ})$ & 11.6 & 0.3 & $10 \cdot 4$ & 0.9 & $9 \cdot 7^{\star \star}$ & 0.9 \\
\hline MUFA (g) & $30 \cdot 8$ & $2 \cdot 9$ & $26 \cdot 0$ & $3 \cdot 1$ & $30 \cdot 1$ & $3 \cdot 7$ \\
\hline MUFA (g/4184 kJ) & $14 \cdot 2$ & 0.2 & $12 \cdot 9$ & 0.9 & $12 \cdot 4$ & 1.0 \\
\hline PUFA (g) & $13 \cdot 4$ & $1 \cdot 2$ & 11.7 & 0.8 & $19 \cdot 3^{\star \star \star}$ & 1.7 \\
\hline PUFA (g/4184 kJ) & $6 \cdot 3$ & 0.06 & $6 \cdot 3$ & 0.35 & 5.5 & 0.6 \\
\hline Linoleic acid $(\mathrm{g})$ & $10 \cdot 7$ & 1.0 & $9 \cdot 3$ & 0.6 & $15 \cdot 8^{\star \star \star}$ & 1.3 \\
\hline Linoleic acid (g/4184kJ) & $5 \cdot 0$ & 0.05 & $5 \cdot 0$ & 0.37 & $7 \cdot 0$ & 1.21 \\
\hline Linolenic acid $(\mathrm{g})$ & 1.9 & 0.2 & $1 \cdot 7$ & 0.2 & $2 \cdot 5^{\star \star \star}$ & 0.2 \\
\hline Linolenic acid (g/4184 kJ) & 0.9 & 0.02 & 0.8 & 0.07 & \multirow{2}{*}{\multicolumn{2}{|c|}{$5 \cdot 3: 1$}} \\
\hline Linoleic:linolenic ratio & \multicolumn{2}{|c|}{$5 \cdot 6: 1$} & \multicolumn{2}{|c|}{$5.5: 1$} & & \\
\hline Cholesterol (mg) & 282 & 27 & 266 & 48 & 285 & 79 \\
\hline Cholesterol (mg/4184kJ) & 138 & 2.4 & 140 & $28 \cdot 1$ & 122 & 20 \\
\hline Vitamin $A(\mu g)$ & 699 & 114 & 613 & 49 & 733 & 130 \\
\hline Vitamin A ( $\mu \mathrm{g} / 4184 \mathrm{~kJ})$ & 357 & 34 & 321 & 21 & 331 & 35 \\
\hline Vitamin C (mg) & 126 & 15 & 150 & 13 & $157^{\star}$ & 14 \\
\hline Vitamin C (mg/4184 kJ) & 66 & $2 \cdot 6$ & $79 \dagger$ & $6 \cdot 6$ & 70 & $6 \cdot 9$ \\
\hline Thiamin (mg) & $1 \cdot 7$ & 0.1 & 1.6 & 0.3 & $2 \cdot 2^{\star \star}$ & 0.3 \\
\hline Thiamin (mg/4184 kJ) & 0.85 & 0.04 & 0.83 & 0.09 & $0.98^{*}$ & 0.05 \\
\hline Riboflavin (mg) & 1.9 & 0.2 & 1.9 & 0.2 & $2 \cdot 2$ & 0.4 \\
\hline Riboflavin (mg/4184 kJ) & 0.96 & 0.01 & 0.96 & 0.05 & 1.00 & 0.1 \\
\hline Niacin $(\mathrm{mg})$ & 40 & 3.5 & 38 & 3.6 & $46 \dagger$ & $6 \cdot 6$ \\
\hline Niacin (mg/4184 kJ) & $19 \cdot 7$ & 0.1 & $19 \cdot 9$ & 1.6 & 20.5 & 0.9 \\
\hline Vitamin $\mathrm{B}_{6}(\mathrm{mg})$ & 1.9 & 0.2 & 1.9 & 0.2 & $2 \cdot 4^{\star \star \star}$ & 0.2 \\
\hline Vitamin $B_{6}(\mathrm{mg} / 4184 \mathrm{~kJ})$ & 0.94 & $0 \cdot 1$ & 0.97 & 0.04 & $1.05^{\star}$ & 0.05 \\
\hline Vitamin $B_{12}(\mu \mathrm{g})$ & 4.4 & 0.2 & 3.9 & 0.7 & $6 \cdot 0$ & $2 \cdot 1$ \\
\hline Vitamin $B_{12}(\mu \mathrm{g} / 4184 \mathrm{~kJ})$ & $2 \cdot 2$ & 0.1 & 1.9 & 0.2 & 2.5 & 0.6 \\
\hline Naturally occurring folate $(\mu \mathrm{g})$ & 234 & 27 & 262 & 25 & $312^{\star \star}$ & 16 \\
\hline Folic acid $(\mu \mathrm{g})$ & 121 & 22 & 123 & 27 & 154 & 42 \\
\hline Folate (from food in dietary folate equivalents) $(\mu \mathrm{g})$ & 462 & 54 & 461 & 24 & 482 & 26 \\
\hline Folate $(\mu \mathrm{g} / 4184 \mathrm{~kJ})$ & 231 & $6 \cdot 9$ & 237 & 8.9 & 219 & $16 \cdot 6$ \\
\hline Vitamin $D(\mu \mathrm{g})$ & $5 \cdot 7$ & 0.4 & $4 \cdot 1$ & 1.0 & $6 \cdot 3$ & 1.4 \\
\hline Vitamin D ( $\mu \mathrm{g} / 4184 \mathrm{~kJ})$ & $2 \cdot 8$ & 0.1 & $2 \cdot 1^{\star \star}$ & 0.3 & $2 \cdot 8$ & 0.4 \\
\hline $\mathrm{Ca}(\mathrm{mg})$ & 855 & 87 & 788 & 115 & $1309^{\star \star}$ & 234 \\
\hline $\mathrm{Ca}(\mathrm{mg} / 4184 \mathrm{~kJ})$ & 423 & 5 & 394 & 42 & $582^{\star \star}$ & 53 \\
\hline$P(m g)$ & 1339 & 113 & 1271 & 112 & $1705^{\star \star}$ & 235 \\
\hline$P(\mathrm{mg} / 4184 \mathrm{~kJ})$ & 658 & 5 & 647 & 17 & $748^{\star \star \star}$ & 23 \\
\hline$M g(\mathrm{mg})$ & 326 & 26 & 336 & 23 & $431^{\star \star \star}$ & 50 \\
\hline $\mathrm{Mg}(\mathrm{mg} / 4184 \mathrm{~kJ})$ & 166 & 3 & $176+$ & 6 & $191^{\star \star \star}$ & $5 \cdot 3$ \\
\hline $\mathrm{Fe}(\mathrm{mg})$ & 14.1 & 0.9 & $13 \cdot 2$ & 0.8 & $19 \cdot 0^{\star \star \star}$ & $2 \cdot 1$ \\
\hline $\mathrm{Fe}(\mathrm{mg} / 4184 \mathrm{~kJ})$ & 7 & 0.3 & $6 \cdot 9$ & 0.4 & $8 \cdot 6^{\star \star \star}$ & 0.3 \\
\hline $\mathrm{Zn}(\mathrm{mg})$ & $11 \cdot 3$ & 0.7 & $11 \cdot 1$ & 0.9 & $13 \cdot 7 \dagger$ & $2 \cdot 0$ \\
\hline $\mathrm{Zn}(\mathrm{mg} / 4184 \mathrm{~kJ})$ & 5.5 & 0.2 & $5 \cdot 6$ & 0.3 & 6.0 & 0.2 \\
\hline $\mathrm{Na}(\mathrm{mg})$ & 3103 & 243 & 2908 & 349 & 3690 & 606 \\
\hline $\mathrm{Na}(\mathrm{mg} / 4184 \mathrm{~kJ})$ & 1530 & 28 & 1493 & 134 & 1655 & 141 \\
\hline $\mathrm{K}(\mathrm{mg})$ & 3114 & 222 & 3118 & 201 & $3579^{\star \star \star}$ & 290 \\
\hline $\mathrm{K}(\mathrm{mg} / 4184 \mathrm{~kJ})$ & 1587 & 36 & 1629 & 73 & 1591 & 91 \\
\hline Grain products (servings) & $5 \cdot 8$ & 0.6 & $5 \cdot 8$ & 0.5 & $6 \cdot 1$ & 0.8 \\
\hline Fruit and vegetable products (servings) & $5 \cdot 2$ & 0.5 & $6 \cdot 1^{\star}$ & 0.6 & $6 \cdot 2$ & 0.4 \\
\hline Milk and milk products (servings) & 1.7 & 0.2 & 1.4 & 0.2 & 1.7 & 0.2 \\
\hline Meat and alternatives (servings) & 4.2 & 0.3 & $4 \cdot 1$ & 0.7 & $5 \cdot 7^{\star \star}$ & $0 . \overline{6}$ \\
\hline
\end{tabular}

Mean value was significantly different from that of non-consumers: ${ }^{*} P<0.05,{ }^{* *} P<0.01,{ }^{* \star *} P<0.001$

† Mean value was different from that of non-consumers $(0.1<P<0.05$; trend). 
in the soya consumer group, is also found in soya beverages $^{(44)}$, with one cup providing nearly one-fourth of the $\mathrm{RDA}^{(45)}$. Tucker's simulation also correctly predicted that replacing meat with soya-based alternatives would lower the intake of saturated fat, which was observed in the present analysis $^{(22)}$. However, it should be noted that the nutrient composition of soya depends dramatically on the type of soya food ${ }^{(46)}$. For example, fresh or dried soyabeans, as well as foods made with fermented soya (miso, tempeh and natto), are more nutrient dense and less processed ${ }^{(47)}$, while soya foods that are processed at a high temperature (soya patties and other soya-based meat alternatives) may have reduced nutrient quality, such as increased $\mathrm{Na}^{(46)}$ as well as lowered levels of isoflavones ${ }^{(46)}$. Data from the CNF show that enriching soya milk adds $250 \mathrm{mg}$ more Ca per serving when compared with its unenriched counterpart ${ }^{(32)}$. Additionally, the quality of soya products is affected by the attributes of the soyabean variety as well as by the environmental conditions in which it is grown. For example, soyabean varieties that are higher in $\mathrm{P}$, protein and fat content produce tofu with higher amounts of these nutrients ${ }^{(48)}$.

In contrast to the concern over the levels of vitamins $B_{6}$ and $\mathrm{B}_{12}$ due to reduced meat consumption, the present analyses reveal that vitamin $B_{12}$ intakes were actually higher among soya consumers and vitamin $\mathrm{B}_{6}$ intake was enhanced among adolescent and adult soya consumers. The reason for this is not apparent, as soya foods are not rich in these vitamins, and further research is required to examine this finding. A possible explanation is that individuals with the highest soya intakes consume approximately one more serving from the meat and alternatives food group, which may account for the higher intake of vitamin $\mathrm{B}_{6}$. Another reason that these nutrients are not compromised in soya consumers may be because they are not replacing dairy and meat products with soya substitutes, but rather consuming them in conjunction with each other. For example, an average soya patty contains $14 \mathrm{~g}$ soya protein $^{(49)}$, which, if compared with the approximately $16 \mathrm{~g}$ soya protein that adult high soya consumers are eating, would probably contribute to the 1.5 more servings of meat and alternatives observed in the present study. However, this can only be speculated because the total amount of soya protein may not be solely attributed to soya-based meat alternatives. The CCHS 2.2 did not account for specific dietary habits or food exclusions (such as vegetarianism). To examine whether soya was consumed as a meat substitute, the $24 \mathrm{~h}$ dietary recall of each respondent was examined for the presence of meat products. The analysis showed that $83 \%$ of soya consumers ate at least one meat product on the day of their dietary recall, confirming that the majority of soya and meat items were consumed together, and not as a substitute.

There are several health implications of these results. Only $40 \%$ of Canadians have reported eating five or more servings of fruits and vegetables per $\mathrm{d}^{(50)}$. The results from the present study showed that Canadian soya consumers exhibited a higher intake of fruits and vegetables. There have also been concerns raised over sufficient intakes of protein, vitamin $\mathrm{B}_{6}$, vitamin $\mathrm{B}_{12}$, vitamin $\mathrm{D}$, riboflavin, $\mathrm{Ca}$ and $\mathrm{Fe}$ in vegan or vegetarians $^{(51-54)}$. Although information on specific diets was not gathered in the CCHS 2.2, intake of these nutrients was enhanced in soya consumers who also tend to be meat consumers, suggesting that soya consumption along with meat appears to improve the nutrient status of the diet. Nonetheless, the higher energy intakes and the increased amount of food eaten among soya consumers may be a cause for concern if this increase in intake is sustained over time. Interestingly, BMI did not differ among consumers and nonconsumers, nor did physical activity status, with similar proportions of respondents in both groups reporting regular physical activity. As such, it remains unclear as to why soya consumers consume more food yet do not weigh more than non-consumers. Future research involving long-term assessment of soya consumption is required to elucidate this trend.

Another potential benefit of soya consumption may be related to bone health. Although studies have shown conflicting effects of soya isoflavones on bone health, intakes of $\mathrm{Mg}$ and $\mathrm{Ca}$ were significantly higher among soya consumers of all age groups. A study in Italy using $3 \mathrm{~d}$ dietary recalls has found that osteoporotic patients had significantly lower levels of these nutrients than controls $(P<0 \cdot 05)$. They showed that $\mathrm{Mg}$ intake greater than $350 \mathrm{mg} / \mathrm{d}$ was correlated with normal bone mineral content $^{(55)}$. In the CCHS 2.2 cohort, soya consumers in the higher intake category attained intakes of $\mathrm{Mg}$ greater than $350 \mathrm{mg} / \mathrm{d}$. Individuals with high soya intakes also consumed more than $1200 \mathrm{mg} \mathrm{Ca}$, a value that has been linked with an increased rate of bone mineralisation in pubertal girls ${ }^{(56)}$ as well as a decreased risk of osteoporosis and diabetes in women $^{(57)}$. In addition, the Framingham Children's Study has observed that children aged $>12$ years who consumed $\geq 2$ servings of dairy products per $d$ coupled with $\geq 4$ servings of meat and alternatives had higher bone mineral content in their later teen years ${ }^{(58)}$, levels of intake that were met by high soya consumers in the age group of 9-18 years. $\mathrm{P}$, which is important for bone health and maintenance, is crucial during puberty due to its role in the growth and formation of bones ${ }^{(59,60)}$, and was found to be higher in the diets of soya consumers.

With respect to CVD, numerous clinical studies have demonstrated the benefits of diets high in fibre and fruits and vegetables and low in saturated $\mathrm{fat}^{(30)}$, a dietary pattern that was evident in soya consumers. However, even the average high soya consumers did not consume sufficient soya protein to derive the protective cardiovascular effect of soya as specified by the FDA health claim that consumption of $25 \mathrm{~g}$ soya protein/ $\mathrm{d}^{(7)}$ and the increased energy intake associated with soya consumption could be deleterious to cardiovascular health.

As the CCHS 2.2 is a cross-sectional survey, a limitation of the present study is that this $1 \mathrm{~d}$ dietary recall may not be a true representation of individual's habitual eating habits, and there is potential for over- or underestimation of soya consumption habits. Additionally, the CCHS 2.2 is a self-reported survey, and non-sampling errors such as non-response, recall bias and social desirability may affect the validity of the results. Although the five-step multiple-pass method utilised during the $24 \mathrm{~h}$ dietary recall has been shown to enhance accuracy and assist the respondent in remembering what and how much food they had consumed ${ }^{(61)}$, it has been reported that the average under-reporting of energy intake in the CCHS 2.2 is estimated 
at $10 \%$, with a greater under-reporting rate being observed among respondents who were overweight or obese, adults compared with teenagers and women compared with men ${ }^{(62)}$. Additionally, frequency of soya consumption cannot be determined. However, this large survey included 35107 individuals, providing greater validity to this type of survey. Information was not collected on specific types of diets (i.e. low carbohydrate, vegetarian or vegan), which may have been useful in further observation of the average Canadian soya consumer. The present study only examined food intake and not the use of supplements among the respondents. Furthermore, the cause-and-effect relationship cannot be assumed for these results, as soya consumption may be a constituent of an overall healthier lifestyle. In addition, there are methodological issues that arise when observing small populations, such as soya consumers. The overall number of consumers is low, and when further split to account for age groups, the resulting estimates may exhibit increased variability and as a result be less efficient ${ }^{(63)}$. Furthermore, it must be cautioned that nutrient content of various soya products may differ by manufacturer, variety and brand. As the present study relied on values from the CNF database, it should be noted that the accuracy of these databases is not always perfect and nutrient composition may vary from product to product.

Soya consumers have higher intakes of fibre and lower intakes of saturated fat, potentially reducing the risk of CVD. They also have higher intakes of $\mathrm{Ca}, \mathrm{Mg}, \mathrm{P}$ and $\mathrm{K}$, which are crucial in preventing bone loss and maintaining bone health $^{(55,57,59)}$. The reasons for the effects of soya consumption on these potential dietary improvements need to be clarified. In particular, the implied increase in energy intake among soya consumers needs to be further explained using long-term studies to determine what dietary habits other than soya consumption are contributing to this pattern.

\section{Acknowledgements}

The authors thank Dr Ian Clara and Kelly Cranswick at the Manitoba Research Data Centre for their help and support in the statistical analysis.

The present study was supported by Manitoba Healthy Living, Seniors and Consumer Affairs, which had no role in the design and analysis of the study or in the writing of this article.

The authors' contributions are as follows: A. N. M., N. Y. and H. M. A. were responsible for the study design; A. N. M. and N. Y. were responsible for the data analyses and interpreting the results; A. N. M., N. Y. and H. M. A. were responsible for the drafting of the manuscript and critical revision. All authors read and approved the final manuscript.

All authors declare that there are no conflicts of interest.

\section{References}

1. Lanou AJ (2011) Soy foods: are they useful for optimal bone health? Ther Adv Musculoskel Dis 3, 293-300.

2. Montgomery KS (2003) Soy protein. J Perinat Educ 12, $42-45$.
3. Messina M (2010) Insights gained from 20 years of soy research. J Nutr 140, 2289S-2295S.

4. Potter SM, Baum JA, Teng H, et al. (1998) Soy protein and isoflavones: their effects on blood lipids and bone density in postmenopausal women. Am J Clin Nutr 68, Suppl. 6, 1375S-1379S.

5. Webb D (2011) Soyfoods made easy - a soy primer. Today's Dietitian 13, 52.

6. National Soybean Research Laboratory (2013) NSRL: National Soybean Research Laboratory. http://www.nsrl.illinois.edu/ index.html (accessed January 2013).

7. Food Labeling: Health Claims; Soy Protein and Coronary Artery Disease (1999) Food and drug administration, HHS: final rule: soy protein and coronary heart disease. Federal Register 64, 57700-57733.

8. Erdman JW Jr (2000) AHA science advisory: soy protein and cardiovascular disease: a statement for healthcare professionals from the nutrition committee of the AHA. Circulation 102, 2555-2559.

9. Sacks FM, Lichtenstein A, Van Horn L, et al. (2006) Soy protein, isoflavones, and cardiovascular health: an American heart association science advisory for professionals from the nutrition committee. Circulation 113, 1034-1044.

10. Zhang X, Shu XO, Gao YT, et al. (2003) Soy food consumption is associated with lower risk of coronary heart disease in Chinese women. J Nutr 133, 2874-2878.

11. Lee H, Lee J, Gourley L, et al. (1991) Dietary effects on breast-cancer risk in Singapore. The Lancet 337, 1197-1200.

12. Kang H, Zhang Y, Yang J, et al. (2012) Study on soy isoflavone consumption and risk of breast cancer and survival. Asian Pac J Cancer Prev 13, 995-998.

13. Trock BJ, Hilakivi-Clarke L \& Clarke R (2006) Meta-analysis of soy intake and breast cancer risk. J Natl Cancer Inst $\mathbf{9 8 ,}$ 459-471.

14. Arjmandi BH, Khalil DA, Smith BJ, et al. (2003) Soy protein has a greater effect on bone in postmenopausal women not on hormone replacement therapy, as evidenced by reducing bone resorption and urinary calcium excretion. J Clin Endocrinol Metab 88, 1048-1054.

15. Messina M \& Messina V (2000) Soyfoods, soybean isoflavones, and bone health: a brief overview. J Ren Nutr 10, 63-68.

16. Wei P, Liu M, Chen Y, et al. (2012) Systematic review of soy isoflavone supplements on osteoporosis in women. Asian Pac J Trop Med 5, 243-248.

17. Park CY \& Weaver CM (2012) Vitamin D interactions with soy isoflavones on bone after menopause: a review. Nutrients $\mathbf{4}$, 1610-1621.

18. Castelo-Branco C \& Cancelo Hidalgo M (2011) Isoflavones: effects on bone health. Climacteric 14, 204-211.

19. Perez-Castrillon JL, de Luis D, Hernandez G, et al. (2009) Isoflavones and bone health. Curr Womens Health Rev $\mathbf{5}$, $125-129$.

20. Weaver CM \& Cheong JM (2005) Soy isoflavones and bone health: the relationship is still unclear. J Nutr $\mathbf{1 3 5}$, 1243-1247.

21. Ashraf H, Schoeppel C \& Nelson J (1990) Use of tofu in preschool meals. J Am Diet Assoc 90, 1114-1116.

22. Tucker KL, Qiao N \& Maras JE (2010) Simulation with soy replacement showed that increased soy intake could contribute to improved nutrient intake profiles in the U.S. population. J Nutr 140, 2296S-2301S.

23. Keinan-Boker L, Peeters P, Mulligan A, et al. (2002) Soy product consumption in 10 European countries: the European Prospective Investigation into Cancer and Nutrition (EPIC) study. Public Health Nutr 5, 1217-1226. 
24. Messina M, Nagata C \& Wu AH (2006) Estimated Asian adult soy protein and isoflavone intakes. Nutr Cancer 55, 1-12.

25. Health Canada (2012) Do Canadian children meet their nutrient requirements through food intake alone? http:// www.hc-sc.gc.ca/fn-an/surveill/nutrition/commun/art-nutrchild-enf-eng.php (accessed January 2013).

26. Health Canada (2012) Do Canadian adults meet their nutrient requirements through food intake alone? http://www. hc-sc.gc.ca/fn-an/surveill/nutrition/commun/art-nutr-adult-eng. php (accessed February 2013).

27. Mudryj AN, Yu N, Hartman TJ, et al. (2012) Pulse consumption in Canadian adults influences nutrient intakes. Br J Nutr 108, Suppl. 1, S27-S36.

28. Health Canada (2012) Canadian Community Health Survey: Cycle 2.2: a guide to accessing and interpreting the data. http://www.hc-sc.gc.ca/fn-an/surveill/nutrition/commun/ cchs_guide_escc-eng.php (accessed January 2013).

29. Statistics Canada (2012) Canadian Community Health Survey, Cycle 2.2 - nutrition common questions and answers for users. http://www23.statcan.gc.ca/imdb-bmdi/pub/document/ 5049_D1_T9_V1-eng.pdf (accessed January 2013).

30. Gidding SS, Dennison BA, Birch LL, et al. (2006) Dietary recommendations for children and adolescents: a guide for practitioners. Pediatrics 117, 544-559.

31. Devaney B, Ziegler P, Pac S, et al. (2004) Nutrient intakes of infants and toddlers. J Am Diet Assoc 104, 14-21.

32. Health Canada (2012) Canadian Nutrient File (CNF). http://webprod3.hc-sc.gc.ca/cnf-fce/index-eng.jsp (accessed December 2012).

33. Health Canada (2013) Canada's food guide: count food servings in a meal. http://www.hc-sc.gc.ca/fn-an/foodguide-aliment/using-utiliser/count-calcul-eng.php (accessed January 2013).

34. United States Department of Agriculture (2013) United States Department of Agriculture Nutrient Data Laboratory. http:// ndb.nal.usda.gov/ (accessed January 2013).

35. Canadian Council on Social Development (2013) Demographics of the Canadian population. http://www.ccSE.ca/ factsheets/demographics/ (accessed January 2013).

36. Population Reference Bureau (2014) Population Reference Bureau. http://www.prb.org/ (accessed January 2014).

37. Statistics Canada (2013) The Chinese community in Canada. http://www.statcan.gc.ca/pub/89-621-x/89-621-x2006001eng.htm (accessed January 2013).

38. Fang CY, Tseng M \& Daly MB (2005) Correlates of soy food consumption in women at increased risk for breast cancer. J Am Diet Assoc 105, 1552-1558.

39. Wenrich TR \& Cason KL (2004) Consumption and perceptions of soy among low-income adults. J Nutr Educ Behav 36, 140-145.

40. Liu Z, Li W, Sun J, et al. (2004) Intake of soy foods and soy isoflavones by rural adult women in china. Asia Pac J Clin Nutr 13, 204-209.

41. Lee M \& Park O (2008) Soy food intake behavior by socio-demographic characteristics of Korean housewives. Nutr Res Pract 2, 275-282.

42. Wansink B \& Chan N (2001) Relation of soy consumption to nutritional knowledge. J Med Food 4, 145-150.

43. Schyver T \& Smith C (2005) Reported attitudes and beliefs toward soy food consumption of soy consumers versus non-consumers in natural foods or mainstream grocery stores. J Nutr Educ Behav 37, 292-299.

44. Kwok KC, Liang HH \& Niranjan K (2002) Optimizing conditions for thermal processes of soy milk. J Agric Food Chem 50, 4834-4838.
45. Institute of Medicine (2012) Dietary reference intakes for thiamin, riboflavin, niacin, vitamin $\mathrm{B}_{6}$, folate, vitamin $\mathrm{B}_{12}$, pantothenic acid, biotin, and choline. http://www.iom.edu/ Reports/2000/Dietary-Reference-Intakes-for-Thiamin-Riboflavin -Niacin-Vitamin-B6-Folate-Vitamin-B12-Pantothenic-Acid-Biotin -and-Choline.aspx (accessed January 2013).

46. Erdman JW Jr, Badger TM, Lampe JW, et al. (2004) Not all soy products are created equal: caution needed in interpretation of research results. J Nutr 134, 1229S-1233S.

47. Slavin JL, Karr SC, Hutchins AM, et al. (1998) Influence of soybean processing, habitual diet, and soy dose on urinary isoflavonoid excretion. Am J Clin Nutr 68, Suppl. 6, 1492S-1495S.

48. Lim B, DeMan J, DeMan L, et al. (1990) Yield and quality of tofu as affected by soybean and soymilk characteristics. calcium sulfate coagulant. J Food Sci 55, 1088-1092.

49. Soyfoods Association of North America (2014) Soy foods protein content chart. http://www.soyfoods.org/nutritionhealth/soy-for-healthy-living/soy-for-heart-disease/soy-proteincontent-chart

50. Statistics Canada (2013) Fruit and vegetable consumption, 2011 http://www.statcan.gc.ca/pub/82-625-x/2012001/article/11661 -eng.htm (accessed January 2013).

51. Craig WJ (2009) Health effects of vegan diets. Am J Clin Nutr 89, 1627 S-1633S.

52. Larsson CL \& Johansson GK (2002) Dietary intake and nutritional status of young vegans and omnivores in Sweden. Am J Clin Nutr 76, 100-106.

53. Nathan I, Hackett AF \& Kirby S (1996) The dietary intake of a group of vegetarian children aged 7-11 years compared with matched omnivores. Br J Nutr 75, 533-544.

54. Tucker KL (2014) Vegetarian diets and bone status. Am J Clin Nutr 100, Suppl. 1, 329S-335S.

55. Tranquilli AL, Lucino E, Garzetti G, et al. (1994) Calcium, phosphorus and magnesium intakes correlate with bone mineral content in postmenopausal women. Gynecol Endocrinol 8, 55-58.

56. Chan GM, Hoffman K \& McMurry M (1995) Effects of dairy products on bone and body composition in pubertal girls. $J$ Pediatr 126, 551-556.

57. Kapetanović A \& Avdić D (2012) Dietary calcium intake and osteoporosis in postmenopausal women living in Sarajevo area. J Health Sci 2, 2.

58. Moore LL, Bradlee ML, Gao D, et al. (2008) Effects of average childhood dairy intake on adolescent bone health. J Pediatr 153, 667-673

59. Kawaura A, Nishida Y \& Takeda E (2005) Phosphorus intake and bone mineral density (BMD). Clin Calcium 15, 1501-1506.

60. Takeda E, Yamamoto H, Yamanaka-Okumura H, et al. (2012) Dietary phosphorus in bone health and quality of life. Nutr Rev 70, 311-321.

61. Moshfegh AJ, Rhodes DG, Baer DJ, et al. (2008) The US department of agriculture automated multiple-pass method reduces bias in the collection of energy intakes. Am J Clin Nutr 88, 324-332.

62. Garriguet D (2008) Under-reporting of energy intake in the Canadian Community Health Survey. Health Rep 19, 37-45.

63. Makvandi E, Bouchard L, Bergeron P, et al. (2013) Methodological issues in analyzing small populations using CCHS cycles based on the official language minority studies. Can J Public Health 104, Suppl. 1, S55-S59. 